\title{
Modeling flux pinning in thin undoped and BazRo3-doped YBCO films
}

\author{
Paturi, P.; Irjala, M.; Huhtinen, H.; Abrahamsen, Asger Bech
}

Published in:

Journal of Applied Physics

Link to article, DOI:

$10.1063 / 1.3066857$

Publication date:

2009

Document Version

Publisher's PDF, also known as Version of record

Link back to DTU Orbit

Citation (APA):

Paturi, P., Irjala, M., Huhtinen, H., \& Abrahamsen, A. B. (2009). Modeling flux pinning in thin undoped and BazRo3-doped YBCO films. Journal of Applied Physics, 105(2), 023904. https://doi.org/10.1063/1.3066857

\section{General rights}

Copyright and moral rights for the publications made accessible in the public portal are retained by the authors and/or other copyright owners and it is a condition of accessing publications that users recognise and abide by the legal requirements associated with these rights.

- Users may download and print one copy of any publication from the public portal for the purpose of private study or research.

- You may not further distribute the material or use it for any profit-making activity or commercial gain

- You may freely distribute the URL identifying the publication in the public portal

If you believe that this document breaches copyright please contact us providing details, and we will remove access to the work immediately and investigate your claim. 


\title{
Modeling flux pinning in thin undoped and $\mathrm{BaZrO}_{3}$-doped $\mathrm{YBCO}$ films
}

\author{
P. Paturi, ${ }^{1}$ a) M. Irjala, ${ }^{1}$ H. Huhtinen, ${ }^{1}$ and A. B. Abrahamsen ${ }^{2}$ \\ ${ }^{1}$ Department of Physics and Astronomy, Wihuri Physical Laboratory, University of Turku, Turku FI-20014, \\ Finland \\ ${ }^{2}$ Materials Research Division, Ris $\phi$ National Laboratory for Sustainable Energy, Technical University of \\ Denmark, P.O. Box 49, DK-4000 Roskilde, Denmark
}

(Received 30 June 2008; accepted 3 December 2008; published online 22 January 2009)

\begin{abstract}
A simple model based on distributions of twin boundaries, dislocations, and $\mathrm{BaZrO}_{3}$ nanorods is presented to describe the $J_{c}$ properties of undoped and $\mathrm{BaZrO}_{3}(\mathrm{BZO})$-doped $\mathrm{YBa}_{2} \mathrm{Cu}_{3} \mathrm{O}_{x}$ thin films. The model accurately describes the shape of $J_{c}(B, T)$ curves of the films, when the pinning site distributions are taken from distributions of twin spacings and BZO nanorods from transmission electron microscope images. Thus, assuming that the model can be used for prediction of the $J_{c}$ properties, we conclude that for enhancement of undoped films more crystalline defects are needed and for doped films a dopant that would create slightly larger rods would be optimal. (C) 2009 American Institute of Physics. [DOI: 10.1063/1.3066857]
\end{abstract}

\section{INTRODUCTION}

In developing superconducting wires for applications, the most important property is the dependence of the critical current density, $J_{c}$, on temperature and magnetic field. Typically, power applications, such as motors, generators, and transformers, need magnetic fields of 3-5 $\mathrm{T}$ with $J_{c}$ $>10^{5} \mathrm{~A} / \mathrm{cm}^{2} .{ }^{1}$ The $J_{c}$ of $\mathrm{YBa}_{2} \mathrm{Cu}_{3} \mathrm{O}_{x}$ (YBCO) wires depends mainly on the microstructure of the superconducting layer. ${ }^{2}$ By inducing suitable flux pinning sites into the lattice, $J_{c}(B, T)$ can be increased substantially. The most effective in pinning are the columnar sites parallel to $c$-axis, which penetrate the whole film. The columnar pinning sites induce angle dependency of $J_{c}$, but in this paper we restrict ourselves to $J_{c}(B \| c)$.

In pure $\mathrm{YBCO}$ films the most obvious film threading pinning sites are the twin boundaries, which are easily observed by transmission electron microscopy (TEM) or x-ray diffraction (XRD). The whole film is covered by twin domains which are a few micrometers long and wide and a single domain is filled by individual twin boundaries with spacing of about $20-30 \mathrm{~nm} .^{3,4}$ The twin planes are oriented evenly in (110) and (110) directions ${ }^{5}$ in films on cubic substrates. The twins are pinning sites for transversal vortex motion and channel vortices along the plane. The overall effect of the twin plane system on $J_{c}$ depends on the microstructure of the twin system, ${ }^{6}$ but in thin films the twin density is high and "dead-end" twins are abundant. The other obvious columnar pinning sites are the threading dislocations on growth island boundaries, ${ }^{7,8}$ but also the twin boundaries are situated there. ${ }^{9}$ The pointlike pinning sites dominating in detwinned single crystals are not nearly as effective as the columnar pinning sites, so they are not taken into account in this paper.

In the last few years doping the $\mathrm{YBCO}$ film with $\mathrm{BaZrO}_{3}$ (BZO) or some other perovskite second phase has been in-

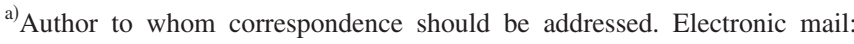
petriina.paturi@utu.fi.
}

tensively studied as a method of increasing the in-field $J_{c}$ of YBCO thin films. ${ }^{4,10-13}$ These second phase nonsuperconducting materials form the so-called nanorods, which are 5-10 $\mathrm{nm}$ in diameter and penetrate the whole film. The most striking effects of the nanorods are forming a wide peak in the $B \| c$ direction in the angular dependency of $J_{c}$ and decreasing the $\alpha$ of the $J_{c} \propto B^{-\alpha}$ behavior.

Even though flux pinning has been extensively studied, a straight link from the experimental results of the structure of the film to $J_{c}$ has been missing. Most of the pinning theories are based at least partly on the extensive treatment of vortices in high-temperature superconductors by Blatter et al. ${ }^{14}$ Recently Pan et al. ${ }^{15}$ published their theory, which links the straight "low angle tilt boundaries" (Laboratories) to $J_{c}(B, T)$. In this paper we present a modification of this theory to twin boundaries, dislocations, and nanorods. We also add temperature dependency to this theory and compare the obtained pinning site distributions to our earlier TEM data $^{4,16}$ on films made with pulsed laser ablation from microand nanograined targets and BZO-doped nanograined targets.

\section{EXPERIMENTAL}

Thin YBCO films were laser ablated on single crystalline $\mathrm{SrTiO}_{3}$ (STO) (001) and MgO (001) substrates using a nanocrystalline target ( $n$-film), a target with micron size grains $\left(\mu\right.$-film) and three different $\mathrm{BaZrO}_{3}$-doped targets (BZO$2 \%-$, BZO-4\%-, and BZO-9\%-films), as described earlier. ${ }^{4,5,17}$ The critical current densities were measured using a superconducting quantum interference device magnetometer at $T=10-70 \mathrm{~K}$ and in $B=0-6 \mathrm{~T}$. The $J_{c}$ 's were determined from the hysteresis loops using the Bean formula for round film samples ${ }^{18,19} J_{c}=3 \Delta M / r$, where the $\Delta M$ is the opening of the hysteresis loop in $\mathrm{A} / \mathrm{m}$ and $r$ is the radius of the sample. The thicknesses of all samples were $150 \mathrm{~nm}$ as determined from atomic force microscopy images taken over an edge chemically etched to the films after the $J_{c}$ measurements.

The microstructure of the films was determined with 

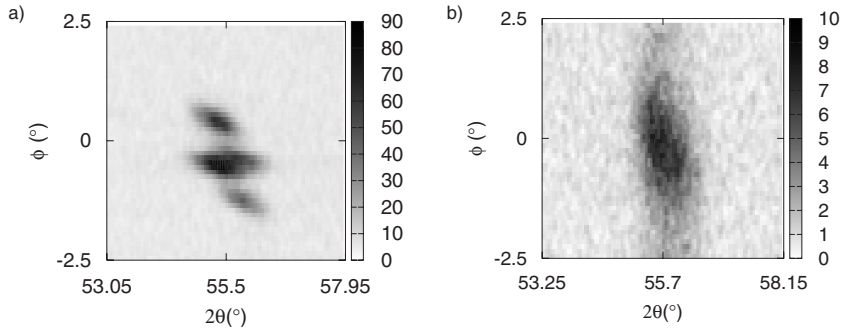

FIG. 1. The XRD $2 \theta-\phi$ scans over the $(122) /(212)$ peak sets of YBCO $n$-films on (a) STO and (b) $\mathrm{MgO}$ substrates.

XRD using a Philips X'pert pro diffractometer with a Schultz texture goniometer. All the films on STO show sharp (212)/(122) peaks with $0.9^{\circ}$ split indicating twins [Fig. 1(a)], whereas the films on $\mathrm{MgO}$ have a broadened $(212) /(122)$ peak set [Fig. 1(b)] caused by low angle grain boundaries, in accordance with earlier TEM data. ${ }^{20}$ The sharp peaks observed in the films on STO, on the other hand, are not compatible with low angle grain boundaries.

For the magneto-optical (MO) microscopy similar samples were patterned to $100 \mu \mathrm{m}$ wide stripes using photolithographic etching. The MO images were taken in the usual Faraday-rotation setup, ${ }^{21}$ where the MO films used were $2.5 \mu \mathrm{m}$ thick liquid phase epitaxial Bi-doped yttrium iron garnet films. An image loop with ascending and descending fields up to $130 \mathrm{mT}$ was recorded for each film at $20-80 \mathrm{~K}$ with $2.5 \mathrm{~K}$ steps. The $J_{c}$ was determined from the last image in the series, i.e., from the remanent field image. The images were enhanced using the routine described in Ref. 22 and the $J_{c}$ calculated using the Fourier method described in Ref. 23, which takes into account the horizontal magnetic field caused by the supercurrents in the film.

TEM was used to measure the distributions of the twin spacings and the BZO distances. The TEM images were taken using a Philips Technai (F20) FEG electron microscope operated at $200 \mathrm{kV}$. To avoid preferential sputtering, the films were ground mechanically using a series of diamond disks and finally thinned with silicon colloidal suspension to electron transparency.

\section{PINNING BY TWIN PLANES}

All YBCO films contain twin planes, which are easily observable by TEM (Ref. 16) and XRD (Ref. 5) [Fig. 2(b)]. The twinning is caused by the tetragonal orthorhombic transition during the cooling of the film from the deposition temperature. In thin films there are a lot more twin planes than in single crystals due to the strain caused by the usually cubic substrate to the orthorhombic YBCO films.

A twin boundary causes an potential well of ${ }^{14}$

$$
\epsilon_{\mathrm{tp}} \approx \epsilon_{0} \Delta,
$$

where $\epsilon_{0}=\phi_{0} /(4 \pi \lambda)$ is the characteristic vortex energy with the flux quantum $\phi_{0}$ and the magnetic penetration depth $\lambda$. $\Delta(T)$ quantifies the relative suppression of the order parameter at the twin planes and is of the order of $10^{-3} .{ }^{14}$ The temperature dependence of Eq. (1) will be treated in Sec. V. The potential energy is counteracted by the elastic energy caused by the vortex lattice
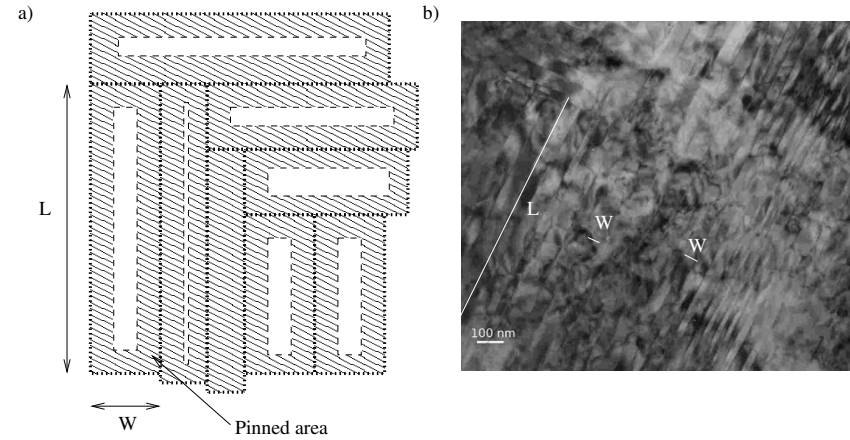

FIG. 2. (a) The pinning model in the twin plane case. The dashed areas show in each case the area where the vortices are pinned and contribute to the $J_{c}$. (b) A TEM image of a $\mu$-film showing the twins. $W$ is the twin spacing and $L$ the length (continues out of the image).

$$
\epsilon_{d}=\frac{\epsilon_{0}}{4 a^{2}} \delta^{2},
$$

where $\delta$ is the displacement of the vortex from its position in the lattice and $a \approx \sqrt{\phi_{0} / B}$ is the distance between the vortices. It is clear from energy conservation ${ }^{15}$ that there is a critical displacement $\delta_{c}$, below which the vortex is pinned to the twin plane. Thus, we get for the pinning radius of the twin boundary the following:

$$
\delta_{\mathrm{c}, \mathrm{tp}} \approx \sqrt{4 \phi_{0} \Delta(T) / B} .
$$

The critical current density can be calculated from considering the probability of the vortex to be within $\delta_{c}$ radius from a pinning site. ${ }^{15}$ This obviously depends on the way the pinning sites are organized in the sample. It should be noted that the $\delta_{c, \mathrm{tp}}$ has the same field dependency as what was obtained for low angle boundaries in Ref. 15.

The areas between the twin planes are elongated rectangles with typical widths of $20-40 \mathrm{~nm}$ and lengths of 2-4 $\mu \mathrm{m}$ (Ref. 16) (see Fig. 2). With good consistency with the experimental data ${ }^{16}$ we can take the twin length, $L$, and spacing, $W$, to follow the gamma distribution as in ${ }^{15}$

$$
P(x ; \nu, \mu)=\frac{\mu^{\nu} x^{\nu-1}}{\Gamma(\nu)} e^{-\mu x},
$$

where $\Gamma(\nu)$ is the gamma function, $\mu$ and $\nu$ are related to the mode of the distribution $M$ and standard deviation of the distribution $\sigma$ as

$$
\begin{aligned}
& \nu=\sigma /(\sigma-M), \\
& \mu=(\sigma-M)^{-1} .
\end{aligned}
$$

The probability of a vortex being in a rectangle with width $W$ and length $L$ between the twin boundaries is

$$
P_{W}(L, W)=\frac{L W P\left(L ; \nu_{l}, \mu_{l}\right) P\left(W ; \nu_{w}, \mu_{w}\right)}{\int_{0}^{\infty} \int_{0}^{\infty} P\left(L ; \nu_{l}, \mu_{l}\right) P\left(W ; \nu_{w}, \mu_{w}\right) L W d L d W},
$$

which is easily derived from area considerations. On the other hand, the probability of the vortex to be within $\delta_{c}$ from the twin boundary is (see Fig. 2) 


$$
P^{\prime}\left(L, W, \delta_{c}\right)=\left\{\begin{array}{cc}
1 & W \leq 2 \delta_{c} \\
1-\frac{\left(L-2 \delta_{c}\right)\left(W-2 \delta_{c}\right)}{L W} & W>2 \delta_{c} .
\end{array}\right.
$$

It is also assumed that $L$ is always much larger than $W$, which is obvious from the TEM images of the twin structures.

The critical current density is proportional to the fraction of pinned vortices to all vortices. ${ }^{15}$ Therefore, we get

$$
\begin{aligned}
\frac{J_{c, \mathrm{tp}}(B, T)}{J_{c}(0, T)}= & \frac{n_{p}}{n_{v}} \approx \int_{0}^{\infty} P_{W}(L, W) P^{\prime}\left(L, W, \delta_{c}\right) d W d L \\
= & 1-\frac{2 \delta_{c} \mu_{l}-\nu_{l}}{\nu_{l} \Gamma\left(1+\nu_{w}\right)}\left[2 \delta_{c} \mu_{w} \Gamma\left(\nu_{w}, 2 \delta_{c} \mu_{w}\right)\right. \\
& \left.-\Gamma\left(1+\nu_{w}, 2 \delta_{c} \mu_{w}\right)\right] .
\end{aligned}
$$

where $\nu_{w}, \mu_{w}, \nu_{l}$, and $\mu_{l}$ are the parameters describing the twin width and length distributions, respectively. It can clearly be seen that since $\mu_{l}$ is about a hundred times smaller than $\mu_{w}$, the twin spacing, $W$, completely dominates the behavior of the vortices.

\section{PINNING BY COLUMNAR DEFECTS}

A columnar defect (cd) in the HTS film causes a potential well with energy depth of ${ }^{14}$

$$
\epsilon_{\mathrm{cd}} \approx-\frac{\epsilon_{0}}{2} \ln \left(1+\frac{r_{0}^{2}}{2 \xi(T)^{2}}\right),
$$

where $\xi(T)=\xi_{0}\left(1-T / T_{\mathrm{c}}\right)^{-1 / 2}$ is the coherence length and $r_{0}$ is the radius of the pinning site. The temperature dependence of Eq. (10) is approximated to be included in the temperature dependence of $\xi$. The potential energy is counteracted by the elastic energy caused by the vortex lattice Eq. (2). Similarly, as for twin planes we get for an arbitrary sized pinning site

$$
\delta_{c} \approx\left[2 \frac{\phi_{0}}{B} \ln \left(1+\frac{r_{0}^{2}}{2 \xi(T)^{2}}\right)\right]^{1 / 2} .
$$

Pure YBCO films contain varying amounts of film penetrating dislocations, ${ }^{8,24}$ which are not randomly situated. Instead the distribution is random only at long distances. ${ }^{25}$ The sizes of these dislocations is $0.3-0.5 \mathrm{~nm}^{20}$ On the other hand, doping YBCO films with BZO leads to $5-10 \mathrm{~nm}$ wide BZO rods to be similarly positioned in the film. ${ }^{4}$ Changing the doping level does not change the size of the rods, only the density of the rods. We model the situation in both kinds of films by inspecting the distances, $l$, from one cd to another (see Fig. 3). The whole sample area can be divided to triangles with cds in the corners, and the probability of the vortex to be pinned is proportional to the fraction of the area covered by the $\delta_{c}$ radii circles from the corners and the total triangle area. The distribution of $l$ depends on the doping and is taken to be the gamma distribution of Eq. (4). The calculation has been done for an equilateral triangle, which is a fairly good approximation as long as the triangles are not very elongated.
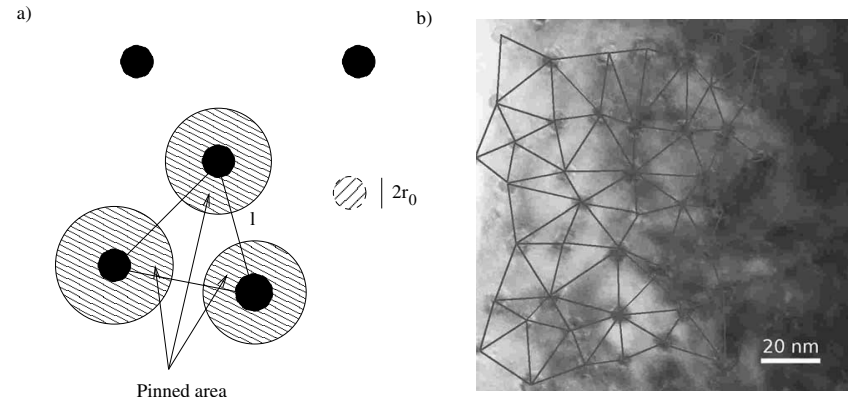

FIG. 3. (a) The pinning model of cds of size $r_{0}$ and separated by the distance $l$. The dashed regions are determined by the critical displacement $\delta_{c}$ where the vortices will be pinned and contribute to the critical current density. (b) A planar TEM image of a BZO-9\%-film showing the BZO rods from top and a possible network of triangles.

With the same approach as above for twin planes, we get that the probability of the vortex to be closer than $\delta_{c}$ to the corner rods is

$$
P^{\prime}\left(x, \delta_{c}\right)=\left\{\begin{array}{cc}
1 & l \leq A \delta_{c} \\
\frac{2 \pi \delta_{c}^{2}}{\sqrt{3} l^{2}} & l>A \delta_{c},
\end{array}\right.
$$

where $A=\sqrt{2 \pi} / 3^{1 / 4}$, which comes from the triangular geometry of the system. Similarly, as above we get for the critical current density

$$
\begin{aligned}
\frac{J_{c, \mathrm{~cd}}(B, T)}{J_{c}(0, T)}= & 1-\frac{1}{\Gamma(\nu+2)}\left[\Gamma\left(\nu+2, A \delta_{c} \mu\right)\right. \\
& \left.-A^{2} d^{2} \mu^{2} \Gamma\left(\nu, A \delta_{c} \mu\right)\right],
\end{aligned}
$$

where $\mu$ and $\nu$ are related to the columnar pinning site distribution mode value $M_{\mathrm{cd}}$ and the standard deviation $\sigma_{\mathrm{cd}}$ in the way shown in Eq. (6).

In reality the BZO-doped films contain both twin planes and BZO rods as pure films contain both twin planes and dislocations. Taking it properly into account would mean modeling both pinning systems together and knowing the relative distributions, which would make the equations unsolvable in closed form. Therefore, we simplify the situation by just adding the two contributions from Eqs. (9) and (13),

$$
\frac{J_{c}(B, T)}{J_{c}(0, T)}=\frac{(1-c) J_{c, \mathrm{~cd}}(B, T)+c J_{c, \mathrm{tp}}(B, T)}{J_{c}(0, T)},
$$

where $0<c<1$ determines the relative weight of the pinning type.

It should be noted that in both models all the parameters, except $\Delta$, are easily comparable to structural properties available from TEM and XRD data. Also, this model gives no estimate of $J_{c}(0, T)$, which should be determined by the depairing current, $j_{0}(0)$, but is generally found to be about a decade lower than $j_{0}(0){ }^{14}$

\section{TEMPERATURE DEPENDENCE OF $J_{c}$}

For determination of $J_{c}(0, T)$ we used $\mathrm{MO}$ imaging, which enables the determination of the local critical current density. The local critical current density strongly depends on the local flux density, the local current path, and the film 


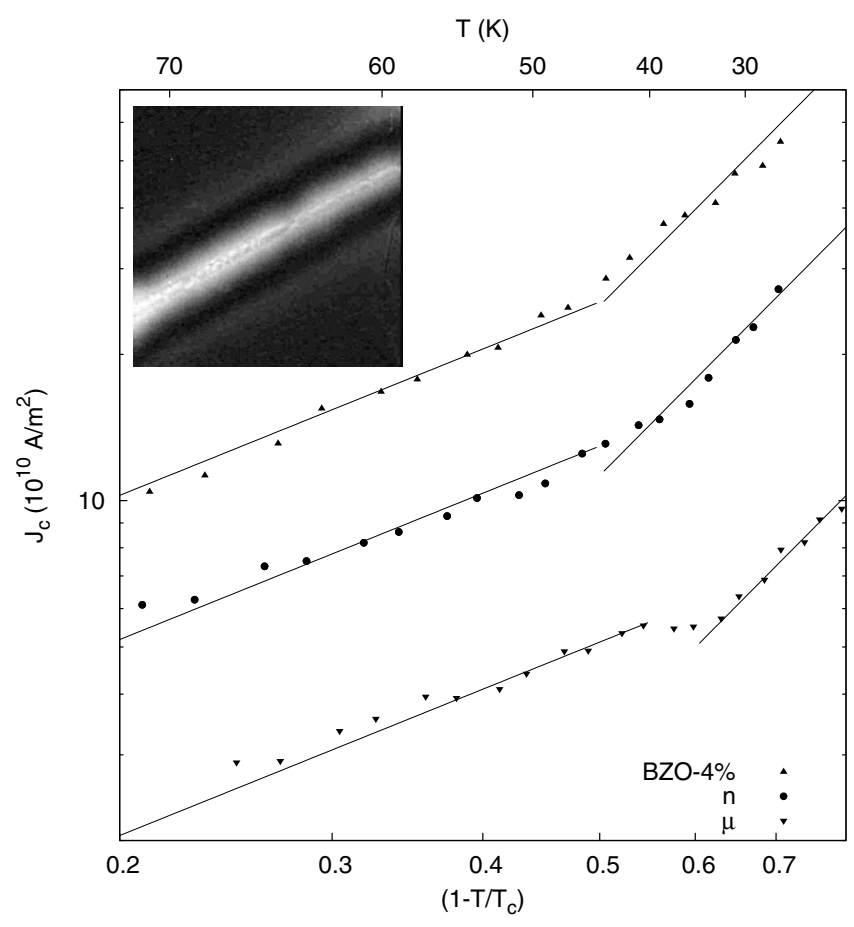

FIG. 4. Temperature dependence of $J_{c}(0, T)$ for the $\mu$-, $n$-, and BZO-4\%films. The lines are the temperature dependences with $\alpha \approx 1$ and $\alpha \approx 2.5$. The inset shows the original MO image for the $\mu$-film at $15 \mathrm{~K}$.

geometry, ${ }^{21}$ which sum up in integrative methods, such as magnetometry or transport critical current measurements. Thus to measure the temperature dependence of the true $J_{c}(0, T)$, one needs to use a local probe, for example, MO imaging. For the determination of $J_{c}$ we chose a region on the films where the current paths were straight and no defects were observed.

Earlier, it has been found that $J_{c}(0, T)$ is proportional to the reduced temperature $\left(1-T / T_{c}\right)^{\alpha}$ with two different $\alpha{ }^{26}$ Since that experiment was done only for undoped YBCO films, we did similar measurements for the $\mu-, n-$, and BZO4\%-films. As can be seen from Fig. 4, the temperature dependence of $J_{c}(0, T)$ is similar for all three films. At temperature $T>40-50 \mathrm{~K}$ the exponent $\alpha \approx 1$ and at $T<40-50 \mathrm{~K}$ $\alpha \approx 2.5$.

For temperature dependence of $J_{c}$ in field, we need to look at the dependence of the potential well depths on temperature. In the first order approximation the potential well caused by the twin planes does not change with temperature as $\Delta$ stays constant up to a high temperature of $\approx 85 \mathrm{~K} .{ }^{14}$ The second order approximation changes the potential well as ${ }^{14}$

$$
\epsilon_{\mathrm{tp}}(T) \approx\left\{\begin{array}{cc}
\epsilon_{\mathrm{tp}} & T<T_{\mathrm{dp}}^{\mathrm{TP}} \\
\epsilon_{\mathrm{tp}}\left(\frac{\tilde{T}_{\mathrm{dp}}^{\mathrm{TP}}}{T}\right)^{2} & T>T_{\mathrm{dp}}^{\mathrm{TP}},
\end{array}\right.
$$

where $T_{\mathrm{dp}}^{\mathrm{TP}}$ is the depinning temperature and $\widetilde{T}_{\mathrm{dp}}^{\mathrm{TP}}$ is the depinning energy, which depends on temperature as ${ }^{14}$

$$
\widetilde{T}_{\mathrm{dp}}^{\mathrm{TP}} \approx \frac{T_{c}}{\sqrt{2 \mathrm{Gi}}} \sqrt{\epsilon_{\mathrm{tp}} / \epsilon_{0}}\left(1-\frac{T}{T_{c}}\right)^{1 / 2},
$$

where $\mathrm{Gi} \approx 10^{-2}$ is the Ginzburg number for YBCO. The depinning temperature is defined as $T_{\mathrm{dp}}^{\mathrm{TP}}=\widetilde{T}_{\mathrm{dp}}^{\mathrm{TP}}\left(T_{\mathrm{dp}}^{\mathrm{TP}}\right){ }^{14}$ These
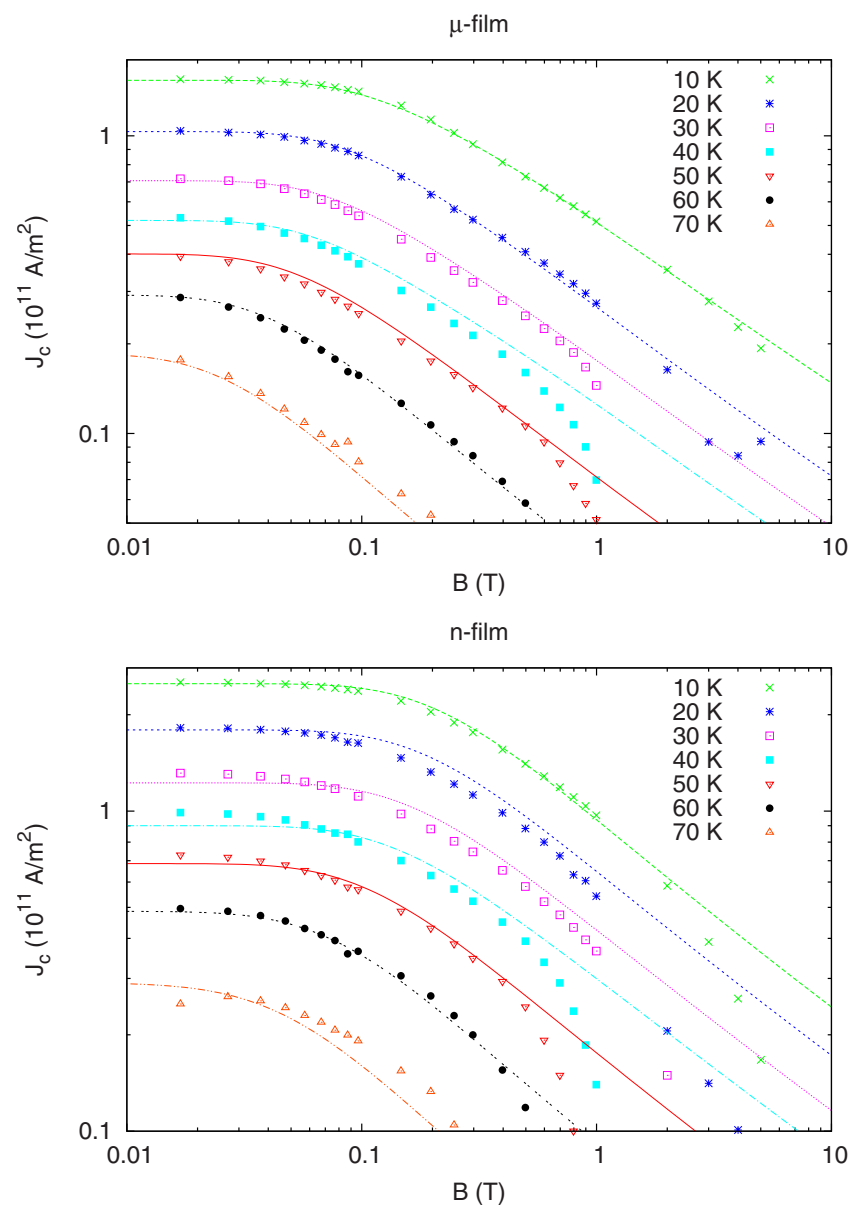

FIG. 5. (Color online) The scaled $J_{c}(T, B)$ for the $\mu$ - and $n$-films on STO and $\mathrm{MgO}$ substrates. The lines are fits to Eq. (14).

can be easily inserted in Eq. (9) for calculations.

The first order approximation for the cd pinning is the temperature dependence of $\xi$ in Eq. (14). We will restrict ourselves to that.

\section{RESULTS}

\section{A. Pinning in undoped YBCO films}

In undoped YBCO films (on STO) the most obvious film threading defects are the twin boundaries, which are always observed. On cubic substrates the twin planes are oriented in two directions with $50 \%-50 \%$ proportion. ${ }^{5}$ On some orthorhombic substrates (such as $\mathrm{NdGaO}_{3}$ ) the twins are oriented in one direction, but they are still there. ${ }^{27}$ Since the twin boundaries channel the vortex motion along them and impede the motion across them, ${ }^{28,29}$ it is obvious that the geometrical size and shape of the twin structure has an effect on $J_{c}$. YBCO films grown on $\mathrm{MgO}$ have variation in the crystal lattice orientation [Fig. 1(b)] seen as widening of the peaks in $\phi$ direction. This is a sign of low angle grain boundaries which probably form around the growth islands, ${ }^{20}$ which is not the case in films on STO. YBCO films on $\mathrm{MgO}$ also usually contain grains which are turned $45^{\circ}$ in the $a / b$-plane.

The $J_{c}(B, T)$ curves for $n$ - and $\mu$-films on STO are shown in Fig. 5. The $J_{c}(B, T)$ curves for the films on $\mathrm{MgO}$ have a slightly different shape from these (not shown); they decrease steeper above $B^{*}$. The main difference between the 
TABLE I. The parameters used in the fits to Eq. (14) for the undoped films. The parameters with errors were fitted; the others were kept fixed in the fitting.

\begin{tabular}{lcc}
\hline \hline Parameter & $\mu$-film & $n$-film \\
\hline$T_{c}(\mathrm{~K})$ & 90 & 90 \\
$\Delta\left(10^{-3}\right)$ & $7.2 \pm 0.8$ & $7 \pm 1$ \\
$M_{w}(\mathrm{~nm})$ & 22 & 22 \\
$\sigma_{w}(\mathrm{~nm})$ & 27 & 29 \\
$M_{l}(\mu \mathrm{m})$ & 2.6 & 2.6 \\
$\sigma_{l}(\mu \mathrm{m})$ & 2.8 & 2.8 \\
$r_{0}(\mathrm{~nm})$ & 0.31 & 0.31 \\
$\nu$ & $9.4 \pm 2.3$ & $8.2 \pm 2.9$ \\
$\mu(1 / \mathrm{nm})$ & $0.19 \pm 0.04$ & $0.24 \pm 0.06$ \\
$M_{\mathrm{cd}}(\mathrm{nm})^{\text {a }}$ & 42 & 29 \\
$\sigma_{\mathrm{cd}}(\mathrm{nm})^{\text {a }}$ & 47 & 33 \\
$c$ & $0.35 \pm 0.03$ & $0.50 \pm 0.06$ \\
& $0.50 \pm 0.01$ for $T=10 \mathrm{~K}$ & \\
\hline
\end{tabular}

${ }^{\mathrm{a}} M_{\mathrm{cd}}$ and $\sigma_{\mathrm{cd}}$ are calculated from $\nu$ and $\mu$ with Eq. (6).

$n$ - and $\mu$-films on STO is the lower $B^{*}$ for the $\mu$-film, which was also seen in Ref. 30. To fit the $J_{c}(B, T)$ curves of the films on STO we used Eq. (14) with the parameters shown in Table I. All the curves at different temperatures were fitted simultaneously. The fitting is worse at high fields and temperatures where flux creep has a significant effect. The errors for the fitting parameters are the standard errors obtained from the nonlinear fitting procedure (Levenberg-Marquardt) and represent a rough estimate, since the assumption of normally distributed measurement errors is not valid (because of, e.g., flux creep).

The only totally free fitting parameters were $\Delta, M_{\mathrm{cd}}, \sigma_{\mathrm{cd}}$, and $c$. The twin width distribution was obtained from the TEM images ${ }^{16}$ and is shown together with the distribution function used in the fit in Fig. 6. As mentioned earlier, the twin length distribution has negligible effect on $J_{c}$, and since the twins are so long, it is difficult to measure the lengths in a statistical way from TEM images, so the length distribution is taken to be the same for both films. The fit gives $\Delta$ of the same order of magnitude than was estimated in Ref. 14 and is almost equal for both films. Approximate size for the dis-
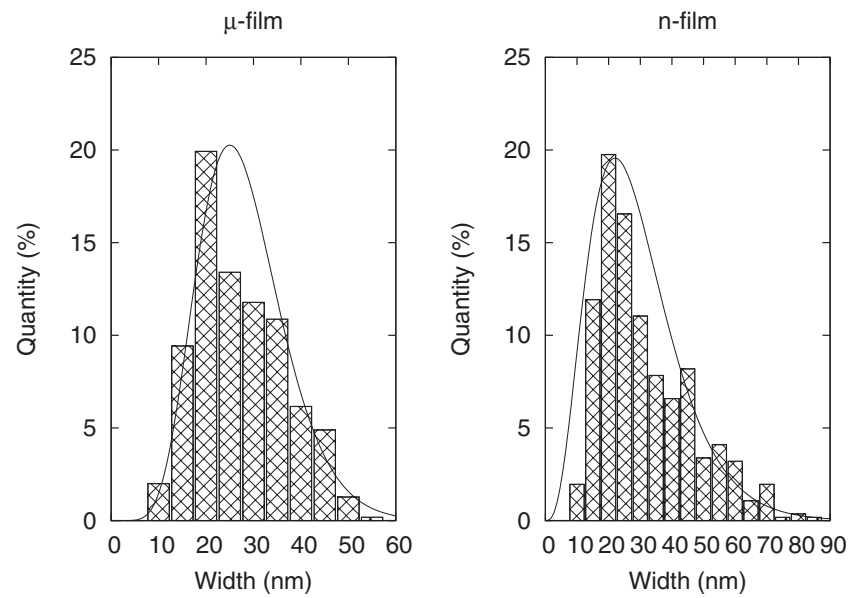

FIG. 6. The twin width distribution measured from the TEM images for undoped $n$ - and $\mu$-films. The lines show the distribution used as fixed parameters in the fits of Fig. 5 . location core $r_{0}$ was taken from Ref. 20 and the $M_{\mathrm{cd}}$ and $\sigma_{\mathrm{cd}}$ were fitted. It can be seen from Table I that the main difference between the $\mu$ - and $n$-films is the distance between the dislocations in the films, which is in perfect agreement with earlier observation of lattice stiffness measured with XRD. ${ }^{16}$ The dislocation densities calculated from the mean distances are 450 and $920 \mu \mathrm{m}^{2}$, which are somewhat larger than what was observed with the etching method. ${ }^{31}$ The densities are still lower than those measured by TEM for films on $\mathrm{MgO} .^{20}$

In the $J_{c}$ of Eq. (14) the weight term $c$ determines the relative proportion of pinning due to twins and dislocations. For $n$-films it is found to be 0.5 , i.e., equal proportion of both pinning systems. For $\mu$-film the best fit for $J_{c}(10 \mathrm{~K})$ was obtained by using $c=0.5$ but at higher temperatures $c=0.35$, i.e., more weight on cds was found better.

\section{B. Pinning in BZO-doped YBCO films}

In BZO-doped films there are two obvious pinning systems: the BZO nanorods and the twin structure. Although the twin structure is not always visible in TEM images, it is clearly present as seen from the splitting of the XRD peaks ${ }^{4}$ and has been seen also in TEM. ${ }^{32}$ In addition, the BZO nanorods are usually surrounded by several dislocations in the YBCO lattice, which also act as pinning sites. ${ }^{16}$ The role of these dislocations is assumed to be covered by the $J_{c, \text { cd }}$ part of Eq. (14).

Equation (14) was used to fit the $J_{c}(B)$ curves in Fig. 7. The fitting parameters, $r_{\mathrm{BZO}}, \Delta$, and $c$ and other used parameters are shown in Table II. Here $r_{\mathrm{BZO}}$ is the effective pinning radius of the BZO rods and the surrounding dislocations. The $M$ and $\sigma$ refer to the mode value and standard deviation of the BZO-rod distance and twin spacing distributions, which were obtained from the TEM images. In Fig. 8 are shown the BZO-rod distance, $l$, and distributions used in the fits along with the distributions measured from TEM images. Unfortunately, since the twin boundaries are not often seen in TEM, we have no statistical information on the width distribution, so we assumed the same twin width distribution as was used in the previous section for undoped $n$-films.

The obtained BZO-rod radii, $r_{\mathrm{BZO}}$, are slightly smaller than the ones measured from TEM images. From Table II it is clear that the differences between the BZO-doped films are explained with the density of the BZO rods, which were obtained from the TEM images. The other difference between the films is the weight of pinning systems in the films. For all films the best fit was obtained when $c$ was allowed to change with temperature. For all the films $c$ increased with temperature indicating that the twin system is more important at high temperatures than the BZO system. In the BZOcontent optimized film (4\%) the change in $c$ was the smallest. Another plausible explanation for the change seen in $c$ is that it is an artifact of the changes in the material parameters (e.g., $\xi$ and $\lambda$ ) of BZO-doped films, which were not allowed change in the fit, or the first order approximation of the temperature dependence of $\delta_{c}$ for cds is not enough and other corrections should be taken into account. 

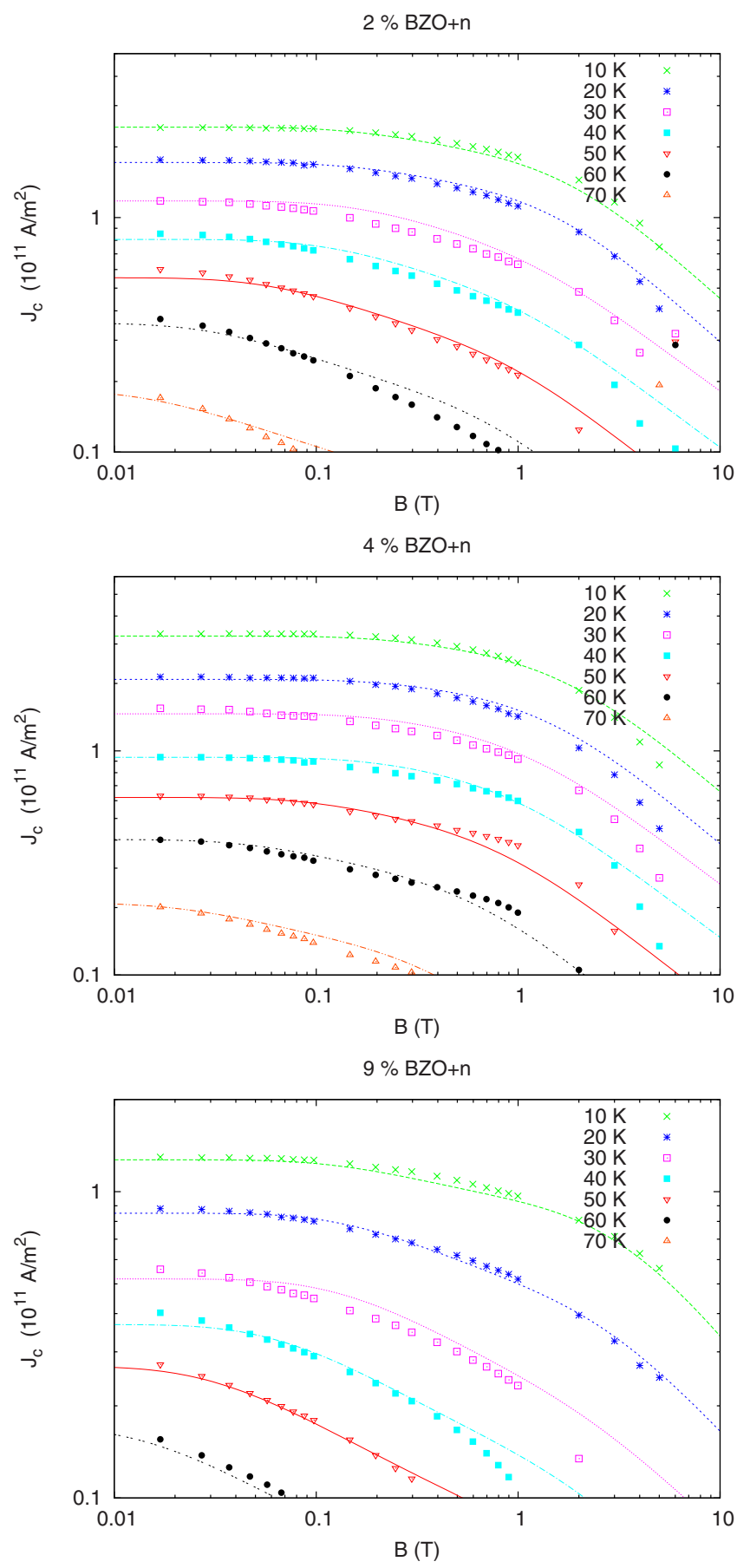

FIG. 7. (Color online) The $J_{c}(B)$ for the $\mathrm{BZO}+n$-films with different doping levels at $5 \mathrm{~K}$. The lines are fits to Eq. (14).

\section{DISCUSSION}

Since the simple model gives surprisingly good results and the parameters are closely related to observed structural properties of the films (twin spacing, BZO-rod distance, and radius), we can make a few observations based on the model. The model, as such, does not predict or even include the contribution to $J_{c}(0, T)$, which should be described with the depairing current $j_{0}(0)$, which theoretically for YBCO is about $2.98 \times 10^{12} \mathrm{~A} / \mathrm{m}^{2}{ }^{33}$ The observed $J_{c}(0, T)$ values from $\mathrm{MO}$ and magnetometer results are about a decade lower than the theoretical $j_{0}(0)$, which is typical for YBCO films and the reason for which is nonperfect pinning in the films.
TABLE II. The parameters used in the fits to Eq. (14) for the BZO-doped films. The parameters with errors were fitted; the others were kept fixed in the fitting.

\begin{tabular}{lccc}
\hline \hline Parameter & $2 \%$ & $4 \%$ & $9 \%$ \\
\hline$T_{c}(\mathrm{~K})$ & 89 & 86 & 81 \\
$r_{\mathrm{BZO}}(\mathrm{nm})$ & $1.15 \pm 0.04$ & $1.10 \pm 0.05$ & $0.90 \pm 0.03$ \\
$M_{\mathrm{BZO}}(\mathrm{nm})$ & 29 & 27 & 20 \\
$\sigma_{\mathrm{BZO}}(\mathrm{nm})$ & 35 & 34 & 25 \\
$\Delta\left(10^{-3}\right)$ & $6.0 \pm 0.3$ & $7.0 \pm 0.4$ & $4 \pm 1$ \\
$M_{\mathrm{tw}}(\mathrm{nm})$ & 22 & 22 & 22 \\
$\sigma_{\mathrm{tw}}(\mathrm{nm})$ & 27 & 27 & 27 \\
$c(T<25 \mathrm{~K})$ & $0.50 \pm 0.05$ & $0.45 \pm 0.05$ & $0.70 \pm 0.06$ \\
$c(T>25 \mathrm{~K})$ & $0.85 \pm 0.04$ & $0.60 \pm 0.02$ & $0.85 \pm 0.06$ \\
\hline \hline
\end{tabular}

The depairing current can only be reached with short current pulses. ${ }^{33}$ Also, the model does not have anything to say about the temperature dependence of $J_{c}(0, T)$ since it only follows the observed dependency. Therefore, the model can only advise us in tuning the magnetic field dependence of the critical current density as dictated by the twin domains and what kind of columnar pinning sites we should aim at to produce YBCO films optimal for certain conditions.

In the undoped YBCO films the main difference between the films was the amount of dislocations in the film. It is clear from the model that if the density of the dislocations increases, $B^{*}$ also increases, as was clearly observed in Refs. 7 and 24. The amount of dislocations can be adjusted with deposition temperature as was done in Ref. 34 or with using a nanocrystalline target. ${ }^{30}$ It was noted in the fitting that the films needed both the twin and the dislocation pinning system to be adequately fitted with the observed values of twin spacing and dislocation core radii. The twin width distribution is even harder to tune than the dislocation density; the mean twin width depends on material parameters, such as twin boundary energy, film-substrate interface stress, and the elastic modulus. ${ }^{3}$ The only parameter that can be affected in deposition is the elastic modulus, which increases with higher concentration of crystalline defects, e.g., dislocations.

The usual method of providing larger columnar pinning sites than what is present in the undoped thin films is doping

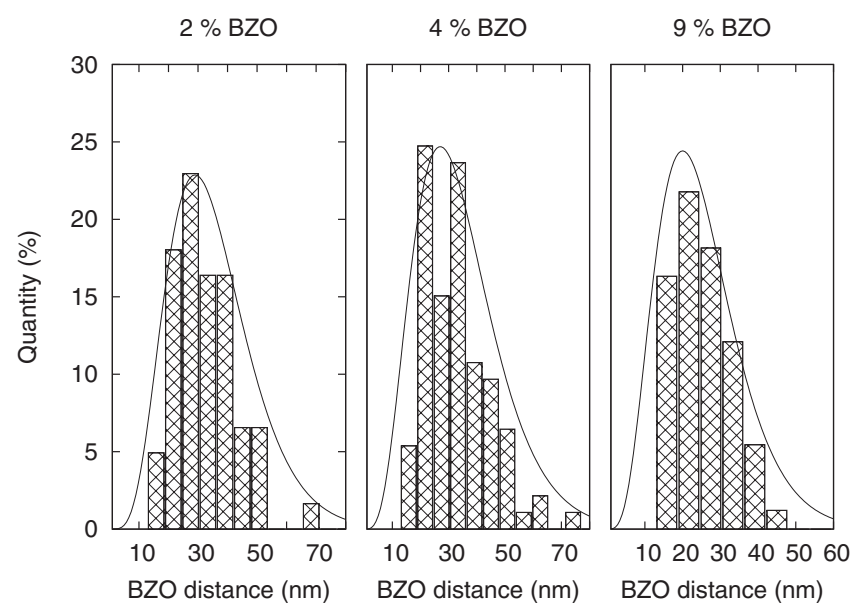

FIG. 8. The BZO rod distance distribution measured from the TEM images. The lines show the distribution used as fixed parameters in the fits of Fig. 7. 


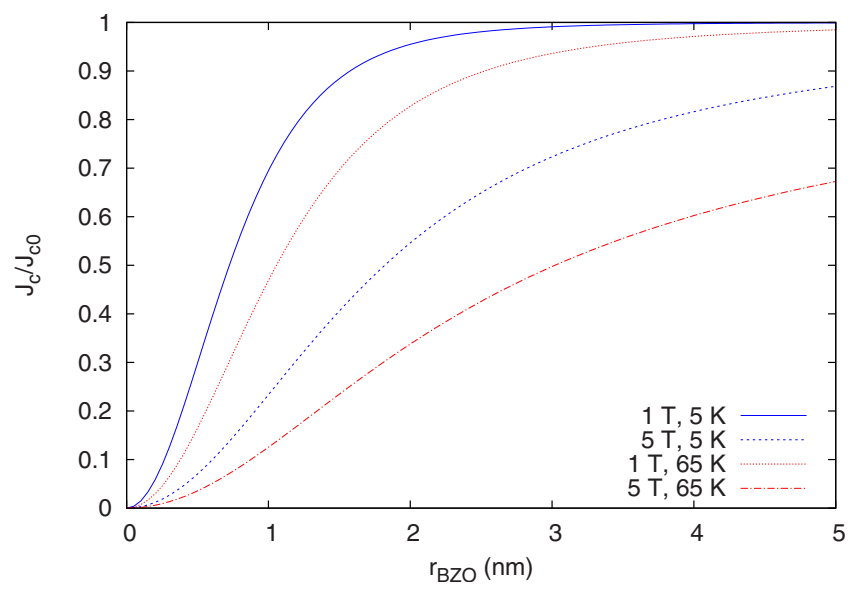

FIG. 9. (Color online) The dependence of the normalized $J_{c}$ on the radius of the rod-line pinning site at different temperatures and magnetic fields. The values are calculated from Eq. (13) using the distribution obtained for the BZO-4\%-film.

the sample with a second phase, such as BZO. The diameter of the rod does not depend on doping level; instead it probably depends the lattice mismatch between the dopant and YBCO. The experimental results for the rod diameters all have similar diameters, ${ }^{4,35}$ but also the lattice mismatches are about the same. According to this model, the optimal $r_{\mathrm{BZO}}$ should be slightly larger than what it currently is (see Fig. 9) for applications at around $5 \mathrm{~T}$. The fit gives $r_{\mathrm{BzO}}$ from the low end of the values observed by TEM; therefore, the optimal 3-4 nm should also be the lower values for the radius of the pinning site. $\mathrm{BaSnO}_{3}$ does provide slightly larger rods than BZO and is also a better dopant at high temperatures, ${ }^{35}$ which agrees well with this model.

In addition to the pinning site radius, the parameter that defines the shape of the $J_{c}(B)$ curve is the shape of the distribution. Very wide distributions lead to gradually decreasing $J_{c}$ with low accommodation field $B^{*}$, whereas very narrow distributions lead to large $B^{*}$ and a very steep decrease in $J_{c}$ above $B^{*}$. Therefore, it is again a question of optimization to decide what kind of distribution is desirable, although $J_{c}$ is a monotonically decreasing function of the mode value and the width of the pinning site distance distribution for both the twin and rod systems. For absolute highest $J_{c}$ it is thus desirable to have as narrow distribution as possible. The limit of adding pinning sites, specially the nonsuperconducting rods, comes from the decrease in $T_{c}$ and $J_{c}(0)$ with high dopings. ${ }^{4}$

\section{CONCLUSIONS}

We have presented a simple model modified from the low angle tilt boundary pinning theory of Pan et al. ${ }^{15}$ to describe the $J_{c}$ properties of undoped and BZO-doped YBCO thin films deposited on STO, where the pinning sites are twin boundaries and BZO nanorods. The model accurately describes the shape of $J_{c}(B, T)$ curves of the films, when the pinning site distributions were taken from distributions of twin spacings and BZO nanorods from TEM images. Thus assuming that the model can be used for prediction of the $J_{c}$ properties, we concluded that for enhancement of undoped films more crystalline defects are needed and for doped films a dopant that would create slightly larger rods would be optimal.

\section{ACKNOWLEDGMENTS}

We thank Dr. Y. Y. Tse from University of Birmingham for the original TEM images and Dr. M. Peurla from University of Turku for the original magnetization measurements of the films, the Academy of Finland, the Wihuri Foundation, the Nordic network for experiments on magnetic and superconducting materials, and the Technical University of Denmark are acknowledged for financial support.

${ }^{1}$ D. Larbalestier, A. Gurevich, D. M. Feldmann, and A. Polyanskii, Nature (London) 414, 368 (2001).

${ }^{2}$ S. R. Foltyn, L. Civale, J. L. MacManus-Driscoll, Q. X. Jia, B. Maiorov, H. Wang, and M. Maley, Nature Mater. 6, 631 (2007).

${ }^{3}$ S. K. Streiffer, E. M. Zielinski, B. M. Lairson, and J. C. Bravman, Appl. Phys. Lett. 58, 2171 (1991).

${ }^{4}$ M. Peurla, P. Paturi, Y. P. Stepanov, H. Huhtinen, Y. Y. Tse, A. C. Bódi, J. Raittila, and R. Laiho, Supercond. Sci. Technol. 19, 767 (2006).

${ }^{5}$ P. Paturi, M. Peurla, K. Nilsson, and J. Raittila, Supercond. Sci. Technol. 17, 564 (2004)

${ }^{6}$ A. Gurevich and L. D. Cooley, Phys. Rev. B 50, 13563 (1994).

${ }^{7}$ B. Dam, J. M. Huijbregtse, F. C. Klaassen, R. C. F. van der Geest, G. Doornbos, J. H. Rector, A. M. Testa, S. Freisem, J. C. Martinez, B. StublePumpin, and R. Griessen, Nature (London) 399, 439 (1999).

${ }^{8}$ J. M. Huijbregtse, B. Dam, R. C. F. van der Geest, F. C. Klaassen, R. Elberse, J. H. Rector, and R. Griessen, Phys. Rev. B 62, 1338 (2000).

${ }^{9}$ G. Kästner, D. Hesse, R. Scholtz, H. Koch, F. Ludwig, M. Lorenz, and H. Kittel, Physica C 243, 281 (1995).

${ }^{10}$ J. L. MacManus-Driscoll, S. R. Foltyn, Q. X. Jia, H. Wang, A. Serquis, L. Civale, B. Maiorov, M. E. Hawley, M. P. Maley, and D. E. Peterson, Nature Mater. 3, 439 (2004)

${ }^{11}$ J. Hänisch, C. Cai, R. Hühne, L. Schultz, and B. Holzapfel, Appl. Phys. Lett. 86, 122508 (2005)

${ }^{12}$ J. Hänisch, C. Cai, V. Stehr, R. Hühne, J. Lyubina, K. Nenkov, G. Fuchs, L. Schultz, and B. Holzapfel, Supercond. Sci. Technol. 19, 534 (2006).

${ }^{13}$ C. V. Varanasi, P. N. Barnes, J. Burke, L. Brunke, I. Maartense, T. J. Haugan, E. A. Stinzianni, K. A. Dunn, and P. Haldar, Supercond. Sci. Technol. 19, L37 (2006).

${ }^{14}$ G. Blatter, M. V. Feigel'man, V. B. Geshkenbein, A. I. Larkin, and V. M. Vinokur, Rev. Mod. Phys. 66, 1125 (1994).

${ }^{15}$ V. Pan, Y. Cherpak, V. Komashko, S. Pozigun, C. Tretiatchenko, A. Semenov, E. Pashitskii, and A. V. Pan, Phys. Rev. B 73, 054508 (2006).

${ }^{16}$ M. Peurla, H. Huhtinen, Y. Y. Tse, J. Raittila, and P. Paturi, IEEE Trans. Appl. Supercond. 17, 3608 (2007).

${ }^{17}$ H. Huhtinen, P. Paturi, E. Lähderanta, and R. Laiho, Supercond. Sci. Technol. 12, 81 (1999).

${ }^{18}$ C. P. Bean, Phys. Rev. Lett. 8, 250 (1962).

${ }^{19}$ L. W. Conner and A. P. Malozemoff, Phys. Rev. B 43, 402 (1991).

${ }^{20}$ V. Svetchnikov, V. Pan, C. Træholt, and H. Zandbergen, IEEE Trans. Appl. Supercond. 7, 1396 (1997).

${ }^{21}$ C. Jooss, J. Albrecht, H. Kuhn, S. Leonhardt, and H. Kronmüller, Rep. Prog. Phys. 65, 651 (2002).

${ }^{22}$ P. Paturi, B. H. Larsen, B. A. Jacobsen, and N. H. Andersen, Rev. Sci. Instrum. 74, 2999 (2003).

${ }^{23}$ P. Paturi, Rev. Sci. Instrum. 76, 093908 (2005).

${ }^{24}$ F. C. Klaassen, G. Doornbos, J. M. Huijbregtse, R. C. F. van der Geest, B. Dam, and R. Griessen, Phys. Rev. B 64, 184523 (2001).

${ }^{25}$ J. M. Huijbregtse, F. C. Klaassen, B. D. R. C. F. van der Geest, and R. Griessen, J. Low Temp. Phys. 117, 663 (1999).

${ }^{26}$ J. Albrecht, M. Djupmyr, and S. Brück, J. Phys.: Condens. Matter 19, 216211 (2007).

${ }^{27}$ C. Villard, G. Koren, D. Cohen, E. Polturak, B. Thrane, and D. Chateignier, Phys. Rev. Lett. 77, 3913 (1996).

${ }^{28}$ R. J. Wijngaarden, R. Griessen, J. Fendrich, and W. K. Kwok, Phys. Rev. B 55, 3268 (1997).

${ }^{29}$ M. V. Indenbom, C. J. van der Beek, M. Konczykowski, and F. Holtzberg, 
Phys. Rev. Lett. 84, 1792 (2000).

${ }^{30}$ M. Peurla, H. Huhtinen, and P. Paturi, Supercond. Sci. Technol. 18, 628 (2005).

${ }^{31}$ J. M. Huijbregtse, B. Dam, R. C. F. van der Geest, F. C. Klaassen, R. Elberse, J. H. Rector, and R. Griessen, Phys. Rev. B 62, 1338 (2000).

${ }^{32}$ P. Mele, K. Matsumoto, T. Horide, A. Ichinose, M. Mukaida, Y. Yoshida, S. Horii, and R. Kita, Supercond. Sci. Technol. 21, 032002 (2008).
${ }^{33}$ W. Lang, I. Puica, K. Siraj, M. Peruzzi, J. Pedarnig, and D. Bäuerle, Physica C 406-462, 827 (2007).

${ }^{34}$ D. M. Feldmann, O. Ugurlu, B. Maiorov, L. Stan, T. G. Holesinger, L. Civale, S. R. Foltyn, and Q. X. Jia, Appl. Phys. Lett. 91, 162501 (2007). ${ }^{35}$ C. V. Varanasi, P. N. Barnes, J. Burke, L. Brunke, I. Maartense, T. J. Haugan, E. A. Stinzianni, K. A. Dunn, and P. Haldar, Supercond. Sci. Technol. 19, L37 (2006). 